\title{
¿OФIA-SOPHIA
}

DOI: http://dx.doi.org/10.18634/sophiaj.14v.2i.793

\section{Alta permanencia de estudiantes en un programa de educación superior*}

\section{Long permanence of students in a higher education \\ program \\ Alta permanência de estudantes em um programa de ensino superior}

Información del artículo

Recibido: Diciembre 13 de 2017 Revisado: Febrero 16 de 2018 Aceptado: Junio 30 de 2018

\section{Cómo citar:}

Pérez, L.M., Muñoz, I., López, A Erazo, A. ,Valencia, A.M., Rojas, V., Viveros, B., Avellaneda, P (2018) Alta permanencia de estudiantes en un programa de educación superior. Sophia, 14 (2), 24-34.
Liliana María Pérez **, Isabel Muñoz ***, Amparo López Higuera**** , Alejandra Erazo*****, Ana María Valencia*****,

Víctor Rojas, Betty Viveros*****, Paola Avellaneda $* * * * *$

* Articulo resultado del proyecto: "Características determinantes de la alta permanencia en los estudiantes matriculados en el Programa de Fonoaudiología de la Universidad del Cauca", desarrollado por el grupo de investigación Comunicación humana y sus desórdenes, Universidad del Cauca, Popayán, Colombia.

**Fonoaudióloga. Especialista en docencia universitaria, profesora titular del Departamento de Fonoaudiología, Facultad Ciencias de la Salud Universidad del Cauca, Popayán, Colombia. Correo de contacto: Imperez@unicauca.edu. co.

***Fonoaudióloga. Magister en Epidemiología, profesora titular Departamento de Fonoaudiología, Facultad Ciencias de la Salud Universidad del Cauca, Popayán, Colombia. Correo de contacto: imunoz@unicauca.edu.co.

****Fonoaudióloga. Magister en Desarrollo Infantil, Especialista en Pedagogía Infantil, Profesora Asistente Departamento de Fonoaudiología, Facultad Ciencias de la Salud Universidad del Cauca, Popayán, Colombia. Correo de contacto: alopezh@unicauca.edu.co.

*****Estudiantes Programa de Fonoaudiología, Facultad Ciencias de la Salud, Universidad del Cauca, Popayán, Colombia.

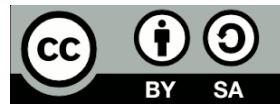

ISSN (electrónico): 2346-0806 ISSN (impreso): 1794-8932 


\title{
Resumen
}

Las instituciones de educación superior se enfrentan a fenómenos que alteran el curso exitoso de las carreras universitarias de los estudiantes, de estos, la alta permanencia trae además de implicaciones económicas para la institución, el estudiante y su familia, un costo emocional alto. Para conocer este fenómeno al interior de la Universidad del Cauca en el Programa de Fonoaudiología, se llevó a cabo un estudio con el objetivo de establecer los determinantes de la alta permanencia de los estudiantes. La metodología fue cuantitativa con alcance descriptivo; se seleccionaron a través de un muestreo aleatorio 107 estudiantes, mediante la revisión del historial académico y se tuvieron en cuenta como criterios de inclusión, estudiantes matriculados que a la fecha del estudio no hubiesen egresado o que aplazaron sus estudios al menos un semestre, logrando una muestra de 60 estudiantes. Los resultados mostraron que la proporción de alta permanencia por sexo fue mayor en el grupo de los hombres, en un 22\% (21), la edad de la población para esta condición, se ubicó entre los 25 y 34 años, correspondiente al 62\% (37). El 65 \% (39) afirmó tener o haber tenido un mal rendimiento académico en el curso de la carrera. Se concluyó que las causas de la alta permanencia estuvieron relacionadas principalmente con el bajo rendimiento académico.

Palabras clave: Estudiantes, factores, permanencia, universidad.

\begin{abstract}
Institutions of higher education face phenomena that alter the successful course of the university careers of students; of these, long permanence brings, besides economic implications for the institution, the students and their families, a high emotional cost. In order to know this phenomenon within the Universidad del Cauca in the Speech Therapy Program, a study was carried out with the objective of establishing the determinants of long permanence of the students. The methodology was quantitative with descriptive scope; 107 students were selected through a random sample by reviewing academic records; as inclusion criteria, there were taken into account enrolled students who at the date of the study had not graduated or who postponed their studies for at least a semester; a sample of 60 students was achieved. The results showed that the proportion of long permanence, by sex, was higher in the group of men, in $22 \%$ (21); the age of the population for this condition was between 25 and 34 years, corresponding to $62 \%$ (37). $65 \%$ (39) claimed to have or have had a poor academic performance in the course of their careers. It was concluded that the causes of long permanence were related mainly to low academic performance.
\end{abstract}

Keywords: Students, factors, permanence, university.

\section{Resumo}

As instituições de ensino superior enfrentam fenômenos que alteram o percurso de sucesso da trajetória universitária dos estudantes, como a longa permanência, que traz consigo implicações econômicas para a instituição, o estudante e sua família e um alto custo emocional. Para conhecer este fenômeno dentro da Universidade do Cauca no Programa de Fonoaudiologia, foi realizado um estudo com o objetivo de estabelecer os determinantes da longa permanência dos estudantes. A metodologia foi quantitativa com escopo descritivo; Foram selecionados 107 estudantes por meio de uma amostra aleatória, revisando o histórico escolar e foram considerados como critérios de inclusão, estudantes matriculados que para o momento do estudo não tivessem se formado ou prorrogado seus estudos por pelo menos um semestre, alcançando uma amostra de 60 alunos. Os resultados mostraram que a proporção de longa permanência por sexo foi maior no grupo de homens, em 22\% (21), a idade da população para essa condição foi entre 25 e 34 anos, correspondendo a $62 \%$ (37). O 65\% (39) afirmou ter ou ter tido um fraco desempenho acadêmico no decorrer da carreira. Concluiu-se que as causas da longa permanência estavam relacionadas principalmente ao mau desempenho escolar.

Palavras - chave: Estudantes, fatores, permanência, universidade. 


\section{Introducción}

Existen diferentes fenómenos educativos que originados por diversas causas alteran el curso exitoso de una carrera universitaria. La deserción, por ejemplo, ha sido un tema ampliamente discutido dentro de la educación superior, esta fue reportada en el año 2010 por el Ministerio de Educación de Colombia (MEN), en un 14\% para el área de ciencias de la salud; además de la deserción, en la realidad educativa universitaria, aparece la alta permanencia o el rezago académico. Este corresponde a la presencia de estudiantes que permanecen en una institución cursando una carrera, más allá del tiempo esperado; en estos casos no hay deserción, pero los estudiantes se rezagan semestre a semestre, haciendo que los años proyectados para la culminación de los estudios se prolonguen.

La alta permanencia en las instituciones de educación superior afecta no solo al sistema académico, sino también al estudiante y a sus familias; especialmente si se considera que la educación universitaria en Colombia debe enfrentar diversos desafíos, entre ellos, los relacionados con la financiación, la autonomía y la calidad de sus procesos administrativos y educativos. Los estudiantes por su parte, deben invertir tiempo y recursos económicos, con el fin de permanecer en el programa que han elegido para cumplir con los objetivos académicos y lograr su graduación.

$\mathrm{Si}$ la eficiencia terminal es entendida como "la relación comparativa entre el número de alumnos que se inscriben por primera vez para cursar una carrera profesional y los de la misma generación que logran egresar" (Camarena, Chávez y Gómez, 1984:1) en el tiempo esperado, la alta permanencia implica que el estudiante demora más tiempo para cumplir con los requisitos curriculares de su programa académico.

Al respecto es importante considerar los factores relacionados con la alta permanencia en los programas académicos, como los personales, académicos, socioeconómicos e institucionales, que determinan en mayor o menor grado la presencia del rezago estudiantil, tal como lo sustenta el (MEN, 2009).

El fenómeno de la alta permanencia se constituye en un tema de gran importancia en la educación superior, considerando las implicaciones en la calidad y la economía que genera. La investigación sobre este tema, es por tanto trascendente considerando que ahonda en el conocimiento sobre las causas que resultan determinantes para que los estudiantes no logren los objetivos curriculares en el tiempo esperado, lo que a su vez aporta a la construcción de estrategias de seguimiento a esta población.

Por esta razón, se realizó una investigación al interior del Programa de Fonoaudiología de la Universidad del Cauca, buscando conocer los factores que incidieron en la alta permanencia de los estudiantes entre los años 1998 y 2012.

\section{Materiales y métodos}

Se realizó un estudio descriptivo de corte transversal, en el Programa de Fonoaudiología de la Universidad del Cauca; con un enfoque social, político y pedagógico. La población estudiada incluyó a todos los estudiantes matriculados a primer semestre entre 1998 y 2012 que atrasaron sus estudios al menos un semestre; los cuales fueron seleccionados mediante la revisión del historial académico disponible en el Sistema de Información, Matricula y Control Académico (Simca).

Con este proceso de selección se obtuvo una muestra inicial de 107 estudiantes que cumplieron con los criterios de inclusión, de los cuales, solo 60 decidieron voluntariamente participar en el estudio y firmar el consentimiento informado. Se aplicó un formato estructurado adaptado de la encuesta de la Asociación Nacional de Universidades e Instituciones de Educación Superior (Romo y Fresan, 2012), después de haber sido sometido a prueba piloto. Este instrumento incluyó preguntas sobre variables sociodemográficas, personales, académicas, familiares y laborales. Además, se realizó un análisis uni y bivariado, a través del paquete estadístico SPSS-19.

\section{Resultados}

Los resultados obtenidos, según los encuestados, mostraron que el bajo rendimiento fue el factor que tuvo mayor incidencia para la alta permanencia. A continuación se presentan los resultados más significativos del estudio.

Los datos sociodemográficos en el estudio describen que el $65 \%$ de los participantes fueron mujeres y el $35 \%$ hombres; el 71\% (43) de ellos solteros. En relación a la edad, el 62\% (37) de los participantes tenían entre 25 y 39 años, y con respecto a la variable procedencia, se observó que el 70\% (42) de los estudiantes eran naturales y residentes en la ciudad de Popayán.

La tabla 1 muestra que la prevalencia de la alta permanencia en el Programa de Fonoaudiología, fue del 30\% representado en 107 estudiantes; de estos solo 
participaron en el estudio el 56\% (60). La proporción de alta permanencia por sexo, para el I periodo académico del año 2013 (356) refleja, que en el grupo de los hombres fue mayor esta situación en un 22\% (21/93), frente a las mujeres que fue del 14\% (39/263).

Tabla 1. Distribución de la prevalencia de la alta permanencia de estudiantes del Programa de Fonoaudiología.

\begin{tabular}{ccc}
\hline Prevalencia total: & Mujeres & Hombres \\
\hline & & \\
Población elegible: $\mathbf{3 0 \%}(107)$ & - & - \\
IC 25,1-34,9 & & \\
& & \\
& $65 \%(39)$ & $35 \%(21)$ \\
Población participante: 16,8\% & IC 53,3- & IC 25- \\
(60) IC 12,8-20,8 & 75 & 46,7 \\
& & \\
Total matriculados al programa & $14 \%$ & $\mathbf{2 2 \%}$ \\
$(356)$ & $(39 / 263)$ & $\mathbf{( 2 1 / 9 3 )}$ \\
& & \\
\hline
\end{tabular}

Fuente: autoría propia

Respecto a la relación de alta permanencia y las variables de origen social y familiar, (tabla 2), se observa que el $86 \%$ de la muestra, percibió su entorno familiar como sano, el $65 \%$ manifestó no haber presentado ninguna calamidad doméstica o problemas de salud. De la población total, el 71\% (43) refirió no haber estado en embarazo ni ser padres, mientras que el $28,3 \%$ sí lo fue.

Tabla 2. Distribución de variables de origen social y familiar en estudiantes con alta permanencia en el Programa de Fonoaudiología.

\begin{tabular}{|c|c|c|c|c|}
\hline \multicolumn{2}{|l|}{ Variables } & \multirow{2}{*}{$\begin{array}{c}\text { N } 60 \\
52\end{array}$} & \multirow{2}{*}{$\begin{array}{c}\% \\
86,7\end{array}$} & \multirow{2}{*}{$\begin{array}{c}\text { IC } 95 \% \\
78,3-95\end{array}$} \\
\hline $\begin{array}{l}\text { Percepción del estudiante } \\
\text { sobre su entorno }\end{array}$ & Sano & & & \\
\hline & Desagradable & 8 & 13,3 & $5-21,17$ \\
\hline & Total & 60 & 100 & \\
\hline Calamidad & Ninguno & 39 & 65 & $52,0-77,9$ \\
\hline \multirow[t]{4}{*}{ y problemas de salud } & Enfermedad & 17 & 28,3 & $16,0-40,5$ \\
\hline & $\begin{array}{l}\text { Muerte de un } \\
\text { familiar }\end{array}$ & 4 & 6,6 & $1,8-16,1$ \\
\hline & Total & & & \\
\hline & & 60 & 100 & \\
\hline \multirow[t]{3}{*}{ Embarazo o paternidad } & Sí & 17 & 28,3 & $16,0-40,5$ \\
\hline & No & 43 & 71,6 & $59,4-83,9$ \\
\hline & Total & 60 & 10 & \\
\hline
\end{tabular}

Fuente: autoría propia 
Tabla 3. Distribución de las variables académicas e institucionales en estudiantes con alta permanencia en el Programa de Fonoaudiología.

\begin{tabular}{|c|c|c|c|c|}
\hline Variables & & n 60 & $\%$ & IC $95 \%$ \\
\hline \multirow{3}{*}{ Institución educativa de bachiller } & & & & $50,3-76,3$ \\
\hline & Público & 38 & 63,3 & $23,6-49,6$ \\
\hline & Privado & 22 & 36,3 & \\
\hline \multirow{4}{*}{$\begin{array}{l}\text { Orientación } \\
\text { profesional }\end{array}$} & Total & 60 & 100 & \\
\hline & Sí & 21 & 35 & $22,3-37,4$ \\
\hline & No & 39 & 65 & $52,09-77,9$ \\
\hline & Total & 60 & 100 & \\
\hline \multirow{5}{*}{$\begin{array}{c}\text { Rendimiento académico } \\
\text { Universitario }\end{array}$} & & & & $20,5-46,0$ \\
\hline & Bueno & 20 & 33,3 & $34,8-61,8$ \\
\hline & Malo & 29 & 48,3 & $7,7-28,9$ \\
\hline & Regular & 11 & 18,3 & \\
\hline & Total & 60 & 100 & \\
\hline \multirow{3}{*}{ Acompañamiento del docente } & Sí & 48 & 80 & $69,09-90,9$ \\
\hline & No & 12 & 20 & $9,04-30,9$ \\
\hline & Total & 60 & 100 & \\
\hline \multirow{4}{*}{$\begin{array}{l}\text { Percepción de la relación docente- } \\
\text { estudiante }\end{array}$} & Bueno & 13 & 21,6 & $10,4-32,9$ \\
\hline & Regular & 25 & 41,6 & $28,3-54,9$ \\
\hline & Malo & 22 & 36,6 & $23,6-49,6$ \\
\hline & Total & 60 & 100 & \\
\hline \multirow{4}{*}{$\begin{array}{l}\text { Percepción del estudiante sobre la } \\
\text { calidad del programa }\end{array}$} & Bueno & 39 & 65 & $52,09-57,9$ \\
\hline & Regular & 20 & 33,1 & $20,5-46,09$ \\
\hline & Malo & 1 & 1,6 & $0,04-8,9$ \\
\hline & Total & 60 & 100 & \\
\hline Insatisfacción & Satisfecho & 40 & 66,6 & $53,9-79,4$ \\
\hline \multirow{2}{*}{ con el programa } & Insatisfecho & 20 & 33,3 & $20,5-46,09$ \\
\hline & Total & 60 & 100 & \\
\hline
\end{tabular}

Fuente: autoría propia 
En la tabla 3 ( ver sig pág ) se presentan los resultados obtenidos en relación a las variables académicas e institucionales, encontrando que la mayor parte de los estudiantes del programa de Fonoaudiología proviene de colegios públicos en un $63 \%$ (38), sin embargo no hay marcada diferencia frente a un $36 \%$, (22) que se graduaron de instituciones privadas. En relación a la orientación profesional que reciben estudiantes previo ingreso a la universidad, se encontró que el 35\% (21) manifestaron haber contado con ella; sin embargo un numero mayor el 65\% (39) de estudiantes, afirmaron no haberla tenido. En cuanto al desempeño académico, la mayor parte de los estudiantes afirmó tener o haber tenido un mal rendimiento académico en el curso de su carrera, en un $48 \%$ (29), seguido del 33\% (20) que lo definieron como bueno, y el restante $18 \%$ (11), lo reportaron como regular; al respecto se pudo terminar que 17 de ellos repitieron 2 asignaturas diferentes, 10 repitieron 4 y 14 han repetido 3 materias distintas.

Con relación al acompañamiento docente el 80\% (48) de los estudiantes afirmó haber contado con este apoyo durante su formación académica e hicieron referencia a aspectos como la forma de evaluar y corregir sus trabajos, contenidos revisados en sus tareas, puntualidad de los docentes en la presentación de los programas de las asignaturas, entrega de resultados de evaluaciones, clases magistrales, pertinencia de temas, claridad en la transmisión de los conceptos, accesibilidad en el trato, acepción a la discusión de sus puntos de vista.

Sobre la percepción del estudiante frente a la relación académica con el docente, el $42 \%$ de los encuestados (25), manifestó haber mantenido una relación regular con sus docentes, siendo este el dato más alto y el $21,6 \%$ de los estudiantes reportó haber tenido una mala relación, y el 22\% (13) la reportaron como buena, en su condición de repitentes.

En lo que respecta a la percepción del estudiante sobre la calidad del programa, el 65\% (39) de estudiantes en condición de alta permanencia calificaron el programa como bueno y el 33\% (20) como regular. Finalmente, el 67\% (40) del total de encuestados manifestaron estar satisfechos con el programa que eligieron, frente a un $33 \%$ (20) que refirieron lo contrario.

La tabla 4 ( ver en pág sig ) muestra los resultados sobre la distribución de los determinantes socioeconómicos y laborales, al respecto se encontró que de la población total, el $91,6 \%$ (55) de los estudiantes dependen económicamente de sus padres, mientras que el 8,3\%, (5) dependen de sus hermanos; es decir, que el 100\% de los estudiantes de la muestra, son económicamente dependientes. Referente a las becas y formas de financiamiento, se encontró que el 96,6\% (58) de los estudiantes en rezago, manifestaron no haber recibido becas o alguna forma para financiar su carrera, frente al 3\% (2), que sí tuvieron este tipo de ayudas. Con relación a la actividad laboral del estudiante, el 71,6\% (43) informó no haber trabajado durante el transcurso de su carrera, mientras que solo el 28,3\% (17) afirmó haber realizado alguna actividad laboral.

Tabla 4. Distribución de las variables socioeconómicas y laborales en estudiantes con la alta permanencia en el Programa de Fonoaudiología

\begin{tabular}{|c|c|c|c|c|}
\hline \multicolumn{2}{|c|}{ Variables } & n 60 & $\%$ & IC $95 \%$ \\
\hline \multirow{4}{*}{$\begin{array}{c}\text { Dependencia } \\
\text { económica }\end{array}$} & $\begin{array}{l}\text { Padres } \\
\text { Hermanos }\end{array}$ & & 91,6 & \multirow{4}{*}{$\begin{array}{c}81,6-97,2 \\
2,7-18,3\end{array}$} \\
\hline & & 55 & 8,3 & \\
\hline & & 5 & 100 & \\
\hline & Total & 60 & & \\
\hline \multirow{4}{*}{$\begin{array}{c}\text { Becas y } \\
\text { formas de } \\
\text { financiamiento }\end{array}$} & Sí & & 3,33 & \multirow{4}{*}{$\begin{array}{c}0,4,-11,5 \\
88,4-99,5\end{array}$} \\
\hline & No & 2 & 96,6 & \\
\hline & & 58 & 100 & \\
\hline & Total & 60 & & \\
\hline \multirow{4}{*}{$\begin{array}{l}\text { Actividad } \\
\text { laboral del } \\
\text { estudiante }\end{array}$} & Sí & & 28,3 & \multirow{4}{*}{$\begin{array}{l}16,0-40,5 \\
59,4-83,9\end{array}$} \\
\hline & No & 17 & 71,6 & \\
\hline & & 43 & & \\
\hline & Total & 60 & & \\
\hline
\end{tabular}

Fuente: autoría propia

Respecto al análisis bivariado, los cruces de variables permitieron encontrar algunos datos significativos:

En la tabla 5 se presenta la relación de sexo con la variable embarazo o paternidad, se encontró que si bien la mayor parte de los estudiantes no enfrentaron estas situaciones, el 33\% (13) de las mujeres se embarazó mientras cursó sus estudios de pregrado. En el caso de los hombres un $19 \%$ (4) enfrentaron la paternidad, lo que suma un $52 \%$ de estudiantes que presentaron alta permanencia y fueron padres.

Sobre la actividad laboral, se encontró que los porcentajes de estudiantes que se dedican a algún trabajo paralelo a sus estudios, son similares entre hombres con $29 \%$ (6) y mujeres con $28 \%$ (11); sin embargo, la mayoría de los estudiantes con alta permanencia no desarrollaban ninguna actividad laboral. 
En relación con el acompañamiento docente con los estudiantes en situación de alta permanencia, se encontró mayor asesoría con las mujeres en un $85 \%$ (33), frente a un $71 \%$ (15) de la realizada con los hombres. Respecto a la calamidad y problemas de salud, son las mujeres quienes presentan mayor frecuencia de enfermedad en un $21,7 \%$ (13), frente a un 6,7\% (4) referido por los hombres. Sin embargo, se presenta un gran número de estudiantes que no refirieron problemas asociados a su salud o situaciones familiares adversas.

Tabla 5. Distribución de estudiantes en alta permanencia según el sexo con las variables de origen social, familiar y laboral.

Respecto a la variable rendimiento académico con relación a la edad, los resultados mostraron que los estudiantes que

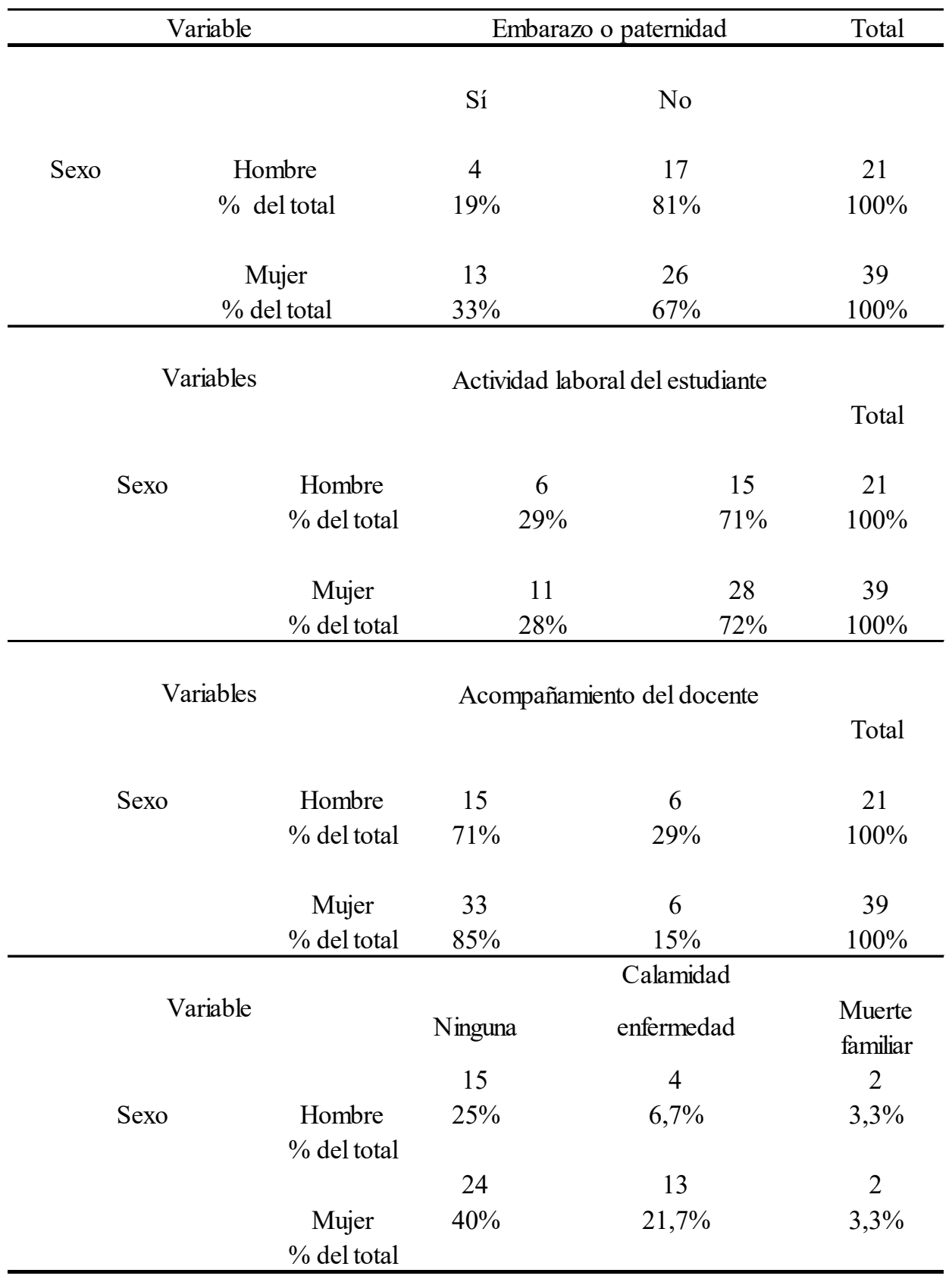

Fuente: autoría propia 
se encuentran en el rango de edad los 25 y 29 años, son los que demuestran rendimiento académico malo y regular; lo que resulta comprensible considerando que en términos de relación entre la edad de matrícula y el progreso de la formación, se muestra rezago y proyección tardía de egreso. También resultó que los estudiantes que refirieron haber solicitado y recibido apoyo del docente, percibieron su rendimiento como malo.

Respecto al cruce de variables de rendimiento académico con las condiciones de trabajo, embarazo o paternidad, calamidad y problemas de salud (tabla 6) no se encontraron relaciones que indicaran como estos últimos guardan una relación porcentual con la alta permanencia; aunque es importante indicar que en casos particulares, condiciones como la maternidad o la muerte de un ser querido representa un bache en el progreso académico semestre a semestre.

Tabla 6. Distribución de estudiantes en alta permanencia según el rendimiento académico con las variables de origen social, familiar y laboral.

\begin{tabular}{llcccc}
\hline & \multicolumn{4}{c}{ Rendimiento académico } & Total \\
\hline & & Bueno & Malo & Regular & \\
Embarazo o & Sí & 5 & 7 & 5 & 17 \\
paternidad & & $8,3 \%$ & $11,7 \%$ & $8,3 \%$ & $28,3 \%$ \\
& No & 15 & 22 & 6 & 71,7 \\
& & $25 \%$ & $36,7 \%$ & $10 \%$ & $60 \%$ \\
Calamidad y & Ninguno & 18 & 18 & 3 & 39 \\
problemas de salud & & $30 \%$ & $30 \%$ & $5 \%$ & $65 \%$ \\
& Enfermedad & 2 & 10 & 5 & 17 \\
& Muerte de & $3,3 \%$ & $16,7 \%$ & $8,3 \%$ & $28,3 \%$ \\
& un familiar & $0 \%$ & $1,7 \%$ & $5 \%$ & $6,7 \%$ \\
Trabajo del & Sí & 6 & 7 & 4 & 17 \\
estudiante & & $10 \%$ & $11,7 \%$ & $6,7 \%$ & 28,3 \\
& No & 15 & 22 & 6 & 43 \\
& & $25 \%$ & $36,7 \%$ & $10 \%$ & $71,7 \%$ \\
Apoyo académico & Sí & 16 & $\mathbf{2 2}$ & 10 & 48 \\
& & $26,7 \%$ & $\mathbf{3 6 , 7 \%}$ & $16,7 \%$ & 80,1 \\
& No & 4 & 7 & 1 & 12 \\
& & $6,7 \%$ & $11,7 \%$ & $1,7 \%$ & $20 \%$ \\
& $20-24$ & 5 & 11 & 2 & 18 \\
& & $28 \%$ & $61 \%$ & $11 \%$ & $100 \%$ \\
Edad en rangos & $25-29$ & 14 & $\mathbf{1 7}$ & $\mathbf{6} \%$ & 37 \\
& & $38 \%$ & $\mathbf{4 6 \%}$ & $\mathbf{1 6 \%}$ & $100 \%$ \\
& \multirow{2}{*}{$20-34$} & 1 & 1 & 3 & 5 \\
& & $20 \%$ & $20 \%$ & $60 \%$ & $100 \%$ \\
\hline
\end{tabular}

Fuente: autoría propia

\section{Discusión de resultados}

La alta permanencia, al igual que sucede con el rendimiento académico, puede explicarse por la: "Interacción entre múltiples capitales en juego" (MEN, 2009:76) entre las que se cuentan los recursos culturales, sociales, sicológicos y materiales; factores que también se han relacionado con el bajo rendimiento y la deserción, lo que permite concluir que hay una estrecha relación entre la alta permanencia y estos otros fenómenos educativos. Los resultados de la investigación realizada en el Programa de Fonoaudiología, dejan ver aspectos de importante análisis como la prevalencia de la alta permanencia que fue del 30\% (107) del total de estudiantes matriculados. 
En estudios realizados por Pinto, Durán, Pérez, Reverón y Rodríguez (2007) y Escobar, Largo y Pérez (2006), se encontró que el $67,8 \%$ y $85,6 \%$ de los estudiantes permanecían matriculados en los programas hasta 4 semestres más de lo establecido en el plan de estudios. En comparación con los resultados del Programa de Fonoaudiología, estos estudios muestran mayores porcentajes de alta permanencia; sin embargo, es importante indicar que el programa matricula regularmente entre 300 y 320 estudiantes, lo que implica que un buen número de estudiantes retrasan su graduación semestre a semestre.

En relación con la edad, el $62 \%$ de la población a estudio superó los 25 años, este hallazgo pone de manifiesto que: en primer lugar, la edad promedio de ingreso a la educación superior en Colombia (Departamento Administrativo Nacional de Estadística [Dane], 2011) es de 17 años, en segunda instancia, el promedio de una carrera universitaria profesional es de aproximadamente 5 años, por lo tanto, quienes ingresan a los 17 años debieran egresar a los 22. Sin embargo, el estudiante que pierde asignaturas o abandona por uno o varios semestres, empieza a ver retrasada su fecha de graduación, lo que hace que la población en rezago estudiantil sea de mayor en edad, que el resto de sus pares.

Si bien la frecuencia absoluta mostró que el $65 \%$ de las mujeres estaban en alta permanencia, la proporción de este fenómeno por sexo mostró que es mayor en los hombres, un 22\% (21/93) frente al 14\% (39/263) de las mujeres. Algunas razones que justifican estos hallazgos son: "Las mujeres parecen adaptarse mejor al ambiente universitario" (Ceballos y Villota, 2003:32) y que incluso en el caso de la deserción los hombres alcanzan tasas más altas frente a las mujeres, dado que según: "Evidencias nacionales e internacionales, coexisten hipótesis sobre aspectos de tipo comportamental y actitudinal de género que definen trayectorias distintas entre hombres y mujeres" (MEN, 2009:93).

Contrario a lo esperado, en el caso del Programa de Fonoaudiología, el mayor número de estudiantes en condición de alta permanencia fueron aquellos cuyo estado civil corresponde a la soltería. Esto resultó contradictorio a lo expresado por Zubieta, Cervantes y Rojas (2009), quienes encontraron que en los solteros había menor rezago académico en comparación con los casados. La explicación a un hallazgo como este, quizá esté determinada por las diferencias que transitan en las poblaciones estudiantiles en la actualidad, estas son distintas en su posición en el contexto social, y en las formas de su subjetividad e identidades conjuntas que permiten que sus características comportamentales divaguen en unos espacios que los circundan y los disipan del sentido habitual en la cultura en la cual están inmersas sus vidas (Belleï, Tenti Fantine y Levinson, 2012). Es decir, las cosas que quizá resultaban importantes y determinantes en la vida de los jóvenes que se enfrentaban a la formación pregradual antes, no tienen la misma representatividad hoy.

Algo similar sucedió con respecto a la percepción del estudiante sobre su entorno familiar, mientras se esperaba que la mayoría la viese como poco positiva, el $86 \%$ (52) de los estudiantes la percibe como sana. Este hallazgo no refleja que la causa familiar sea un determinante en el rezago o alta permanencia para el Programa de Fonoaudiología. En este caso, es probable que si bien hay apoyo familiar, este no se constituye en un determinante considerando los cambios sobre las formas de ver la vida: "Los estudiantes son otros, en su posición en el espacio social y en los contextos familiares" (Belleï, Tenti Fantine y Levinson, 2012:13). A esto se suma que muchas familias pueden incorporar en su discurso la importancia de la preparación académica, pero trasmiten a sus hijos de manera indirecta un cierto capital cultural y algunos ethos, que pueden indicar lo contrario (Bourdieu como se citó en Belleï, Tenti Fantine y Levinson, 2012 ).

A pesar del menor porcentaje $(13 \%)$ de quienes percibieron su entorno familiar como poco positivo, vale la pena mencionar que en este grupo particular, puede haber una estrecha relación entre la percepción del ambiente familiar, su dinámica y el cómo entienden los padres la importancia del estudio con los resultados académicos (Torres y Rodríguez, 2006), en este sentido el medio familiar puede ser un amortiguador del estrés, o ayudar a afrontar la transición y la permanencia en la universidad (Mori, 2012), no contar con él, en muchos casos, puede ser determinante para los logros académicos.

En cuanto a la presencia de adversidades (calamidad familiar) y problemas de salud, se encontró que el $65 \%$ (39) de los encuestados con alta permanencia, manifestaron no haber sufrido ninguna de estas durante el transcurso de su carrera, solo el $28 \%$ reportaron haber padecido alguna enfermedad. Así, para el Programa de Fonoaudiología, estas variables parecen no aportar al fenómeno de alta permanencia. 
En relación con la variable embarazo o paternidad, se encontró que de la población con rezago académico, el $72 \%$ (43) de estudiantes no se enfrentaron a esta condición; sin embargo, para quienes sí estuvieron en esta situación, este fue un motivo para retrasar al menos por un semestre sus estudios. Al respecto, Arévalo Rodríguez (2007) al investigar sobre las razones por las que estudiantes demoran su graduación, encontró que una de estas fue el embarazo, ocupando el cuarto lugar de las diez causas más frecuentes para la alta permanencia.

Referente al rendimiento académico, el $48 \%$ y el $18 \%$ lo percibieron como malo y regular, lo que es natural considerando que son estudiantes que pierden cursos o retrasan su egreso. Sobre este particular, Vera-Noriega, Ramos-Estrada, Sotelo-Castillo, Echeverría-Castro, Serrano-Encinas y Vales-García (2011), también encontraron un alto porcentaje de estudiantes en bajo rendimiento como un elemento asociado al rezago, añaden que es la reprobación acumulada de materias, la que se asocia directamente con el rendimiento académico, lo que pone en relevancia los factores académicos como causa del retraso en el egreso del programa académico matriculado.

En cuanto a la satisfacción con el programa, se encontró que el $67 \%$ estaba complacido con su elección, lo que explica por qué a pesar de los reveses académicos, los estudiantes no abandonaron sus estudios. Cabe señalar que hay aceptación de la propuesta de formación del programa por parte de los estudiantes; sin embargo, los aspectos relacionados con el rendimiento académico, generan interrogantes sobre las estrategias metodológicas que los docentes usan para la formación.

\section{Conclusiones}

Los resultados de este estudio permiten concluir que la edad promedio de los estudiantes con rezago, se encuentra en el rango entre los 24 y 29 años, por lo que es visible cómo el retraso en la graduación prorroga la salida al mercado en casi cuatro años en algunos casos.

Los factores que parecen relacionarse de manera importante con la prolongación de los estudios en el Programa de Fonoaudiología, fueron el bajo rendimiento académico y la falta de orientación profesional, pero se esperaba una presencia de más factores; esto supone que hay una estrecha relación entre la alta permanencia y otros fenómenos culturales, psicológicos y educativos que pueden influir.
No se evidenció que los determinantes socioeconómicos y laborales en los estudiantes en condición de rezago estudiantil, pudieran haber incidido en esta situación; así como tampoco influyeron condiciones de maternidad, paternidad, problemas de salud o el ambiente laboral. Lo anterior permite concluir que si bien los resultados se manifiestan en una posición contraria a lo esperado, en la actualidad entra en juego la teoría de la interacción múltiple y los desafíos culturales y familiares, que implican nuevos comportamientos y conductas de los estudiantes.

La alta permanencia se vio más influenciada por el bajo rendimiento académico en los estudiantes que cursaban IV, V y VI semestre, en los que se inicia el curso de las asignaturas disciplinares. A partir de VI semestre en adelante, se evidencia una estabilidad en un alto porcentaje, con tendencia constante, en los semestres VII, VIII, IX y X semestre, lo que coincide con los hallazgos de otros estudios.

Los estudiantes en condición de alta permanencia, demoraron aproximadamente 8 años para culminar un plan de estudios que dura 5 , aspecto que limita el potencial laboral y cumplimiento de indicadores de calidad académica.

Es fundamental para el Programa de Fonoaudiología, realizar la indagación cualitativa de la percepción de los estudiantes con respecto a sus dificultades académicas y otros factores implicados, con el fin de elaborar un plan de seguimiento que contribuya a reducir este fenómeno.

\section{Agradecimientos}

Los autores expresan sus agradecimientos a la Universidad del Cauca y a los estudiantes del Programa de Fonoaudiología que participaron en el estudio y a los que hicieron parte del desarrollo de esta investigación.

\section{Declaración de conflicto de intereses}

Los autores manifiestan ser independientes y declaran no tener ningún conflicto de intereses.

\section{Referencias bibliográficas}

Arévalo Rodríguez, I. (2007). Caracterización de la problemática de alta permanencia en carreras de pregrado de la Universidad Pedagógica Nacional. Universidad Pedagógica Nacional, Bogotá, Colombia. 
Belleï, C; Tenti Fanfani, E y Levinson, B. A. (2012). La escoLarización de los adoLescentes: desafios cuLturaLes, pedagógicos y de poLítica educativa. Organización de las Naciones Unidas para la Educación, la Ciencia y la Cultura, Instituto Internacional de Planeamiento de la Educación, Sede Regional Buenos Aires.

Camarena, R. M., Chávez, A. M y Gómez, J. (1984). Eficiencia terminal en la UNAM: 1970-1981. Revista Perfiles Educativos, 7, $35-48$.

Ceballos, M. y Villota, D. Factores asociados a la deserción estudiantil en la cohorte 2003 periodo B del Programa de Licenciatura en educación Básica con énfasis en humanidades. (Tesis de pregrado). Universidad de Nariño, San Juan de Pasto, Colombia.

Departamento Administrativo Nacional de Estadística. (2011). Estadísticas vitales 2011. Recuperado de: http://www.bvs.org.ar/pdf/anuario11.pdf.

Escobar, J., Largo, E y Pérez, C. A. (2006). Factores asociados a la deserción y permanencia estudiantil en la Universidad del Valle (1994-2006). Cali, Universidad del Valle, Vicerrectoría Académica, Facultad de Ciencias Sociales y Económicas, Centro de Investigación $y$ documentación socioeconómica-CIDSE.

Ministerio de Educación Nacional. (2009). Deserción estudiantil en la Educación Superior Colombiana. Recuperado de: $\quad$ http://www.mineducacion.gov.co/ sistemasdeinformacion/1735/articles-254702 libro desercion.pdf.

Ministerio de Educación Nacional. (2010). Ingreso, permanencia y graduación. Recuperado de: http://www.mineducacion.gov.co/1621/ articles-92779 archivo pdf_Boletin14.pdf.

Mori, M. (2012). Deserción universitaria en estudiantes de una universidad privada de Iquitos. Revista Digital de Investigación en Docencia Universitaria (RIDU).

Pinto, M., Durán, D., Pérez, R., Reverón, C y Rodríguez, A. (2007). Cuestión de supervivencia. Graduación, deserciónyrezagoenlaUniversidad Nacional de Colombia. Universidad Nacional de Colombia. Dirección Nacional de Bienestar Universitario, Bogotá.
Romo, A y Fresán, M. (2012). Los factores curriculares $\mathrm{y}$ académicos relacionados con el abandono y el rezago. Deserción, rezago y eficiencia terminal en las IES, propuesta metodológica para su estudio. México: ANUIES.

Torres, L. y Rodríguez, N. (2006). Rendimiento académico y contexto familiar en estudiantes universitarios. Enseñanza e investigación en psicología, 11(2), 255-270.

Universidad del Cauca. (2011). Boletín estadístico. Recuperado de: http://www.unicauca.edu.co/ versionP/boletin-estadistico.

Vera-Noriega, J. Á., Ramos-Estrada, D. Y., Sotelo-Castillo, M. A., Echeverría-Castro, S., Serrano-Encinas, D. M y Vales-García, J. J. (2012). Factores asociados al rezago en estudiantes de una institución de educación superior en México. Revista iberoamericana de educación superior, 3(7), 41-56.

Zubieta, G; Cervantes, P y Rojas, S. (2009). La deserción y el rezago en la Educación Superior a Distancia: signos promisorios en una Universidad Pública Mexicana. Observatorio de la Educación Virtual en América Latina y el Caribe. 